\title{
Time-reversed optical parametric oscillation
}

\author{
Stefano Longhi \\ Dipartimento di Fisica, Politecnico di Milano, Piazza L. da Vinci 32, I-20133 Milano, Italy
}

\begin{abstract}
In recent works, the idea of time-reversed laser oscillation has been proposed and demonstrated to realize a two-channel coherent perfect absorber [Y.D. Chong et al., Phys. Rev. Lett. 105, 053901 (2010); W.Wan et al., Science 331, 889 (2011)]. Here the time reversal of optical parametric oscillation in a nonlinear $\chi^{(2)}$ medium is considered and shown to realize a coherent perfect absorber for colored incident signal and idler fields. A detailed analysis is presented for the time-reversed process of mirrorless optical parametric oscillation in the full nonlinear regime.
\end{abstract}

PACS numbers: 42.65.Yj, 42.65.Ky, 42.65.Sf

Introduction. Time-reversal symmetry plays an important role in classical electromagnetic theory and nonrelativistic quantum mechanics [1]. Such a symmetry implies that, if a particular physical process is allowed, then there also exists a time-reversed process, which is obtained by changing the sign of the time variable in the dynamical equations. In recent works, the time-reversed process of laser oscillation has been explored, both theoretically [2] and experimentally [3], and shown to realize a coherent perfect absorber (CPA). Under additional symmetry constraints, the processes of lasing and CPA can even occur simultaneously, and a CPA-laser device can be in principle realized [4, 5]. Since in a steady-state process time reversal corresponds to interchanging incoming and outgoing fields, the time-reversed process of a laser instability corresponds to perfect absorption of incoming light fields. Such a phenomenon is rather general and expected to occur for the time reversal of other optical instabilities. Among these, optical parametric oscillation (OPO) provides an important example of instability in nonlinear optics, which is at the core of the operation of such important devices as optical parametric oscillators [6 9]. In a parametric oscillator at the onset of instability, a pump field at frequency $\omega_{3}$ propagating in a nonlinear $\chi^{(2)}$ crystal generates down-converted fields at frequencies $\omega_{1}$ (the signal field) and $\omega_{2}=\omega_{3}-\omega_{1}$ (the idler field), provided that the phase matching (momentum conservation) condition is satisfied. Generally, at least one of the signal or idler waves resonates in an optical cavity. In the time-reversed process, exactly the opposite occurs: two coherent signal and idler fields, with appropriate amplitude and phase relationship incident onto the pumped nonlinear crystal, are completely annihilated, thus realizing a kind of "colored" CPA device. While a CPA based on time reversal of a laser usually converts the absorbed light into heat 2, 3, in the time reversal of OPO the annihilated photons are converted into light of different frequency (the sum-frequency field). Moreover, since the OPO instability and its time-reversal process are allowed in the same medium, an ordinary OPO device can behave at the instability like a CPA-laser device [4, 5], i.e. it can simultaneously emit outgoing colored fields and absorb incoming colored fields with appropriate phase relationships. This important property shows that a conventional OPO system at threshold is a CPA-laser of a certain kind. In this Letter we consider specifically the time-reversed process of OPO for counterpropagating photons, i.e. the time-reversed process of backward OPO [8, 10, 11, which enables a simple analytical study. Backward OPO describes an interesting three-wave interaction process in a quadratic medium which has the unique property of automatically establishing distributed feedback, thus allowing mirrorless OPO. Mirrorless OPO was theoretically predicted in the early days of nonlinear optics [10, however its experimental demonstration has been reported only in recent years using short-period quasi-phase-matching (QPM) nonlinear crystals [12, 13]. Backward parametric interaction is also useful for the generation of entangled twin photons [14.

Time-reversal of OPO instability: general aspects. We consider interaction of three quasi-monochromatic waves at carrier frequencies $\omega_{1}$ (signal field), $\omega_{2}$ (idler field) and $\omega_{3}=\omega_{1}+\omega_{2}$ (pump field) in a nonlinear $\chi^{(2)}$ medium in the framework of classical nonlinear optics equations. In the scalar approximation, the electric field $\mathcal{E}(\mathbf{r}, t)$ satisfies the wave equation $\nabla_{\mathbf{r}}^{2} \mathcal{E}-\left(1 / c_{0}^{2}\right) \partial_{t}^{2} \mathcal{E}=$ $\left(1 / \epsilon_{0} c_{0}^{2}\right) \partial_{t}^{2} \mathcal{P}$, where $c_{0}$ is the speed of light in vacuum, $\epsilon_{0}$ is the vacuum permittivity, $\nabla_{\mathbf{r}}^{2}$ is the spatial Laplacian, and $\mathcal{P}=\mathcal{P}_{L}+\mathcal{P}_{N L}$ is the polarization of the medium, which includes the linear $\left(\mathcal{P}_{L}\right)$ and nonlinear $\left(\mathcal{P}_{N L}\right)$ terms. For nonlinear interaction in a quadratic medium with QPM, one can assume $\mathcal{P}_{N L}=$ $\epsilon_{0} \chi^{(2)} \mathcal{E}^{2}$, where $\chi^{(2)}(\mathbf{r})$ is the spatially-modulated second-order susceptibility of the medium. After setting $\mathcal{E}(\mathbf{r}, t)=(1 / 2)\left[E_{1}(\mathbf{r}, t) \exp \left(i \omega_{1} t\right)+E_{2}(\mathbf{r}, t) \exp \left(i \omega_{2} t\right)+\right.$ $\left.E_{3}(\mathbf{r}, t) \exp \left(i \omega_{3} t\right)+c . c.\right]$, where $E_{l}(\mathbf{r}, t)$ varies slowly with respect to time $t$ over one oscillation cycle $2 \pi / \omega_{l}(l=$ $1,2,3)$, neglecting group velocity dispersion and indicating by $\epsilon_{l}(\mathbf{r})$ the relative dielectric constant of the medium at the frequency $\omega_{l}$, the envelopes $E_{l}$ satisfy the coupled wave equations (see, for instance, [15])

$$
\begin{gathered}
\left(\nabla_{\mathbf{r}}^{2}+\frac{\omega_{1,2}^{2} \epsilon_{1,2}}{c_{0}^{2}}-\frac{2 i k_{1,2}}{v_{1,2}} \partial_{t}\right) E_{1,2}=-\frac{\omega_{1,2}^{2}}{c_{0}^{2}} \chi^{(2)} E_{2,1}^{*} E_{3} \\
\left(\nabla_{\mathbf{r}}^{2}+\frac{\omega_{3}^{2} \epsilon_{3}}{c_{0}^{2}}-\frac{2 i k_{3}}{v_{3}} \partial_{t}\right) E_{3}=-\frac{\omega_{3}^{2}}{c_{0}^{2}} \chi^{(2)} E_{1} E_{2}
\end{gathered}
$$

where $k_{l}=\omega_{l} n_{l} / c_{0}, n_{l}$ and $v_{l}(l=1,2,3)$ are the wave 


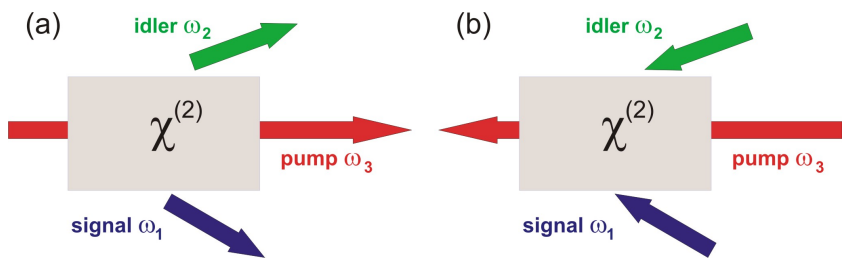

FIG. 1. (Color online) Schematic of (a) an OPO instability, and (b) its time-reversed process, corresponding to a colored CPA.

number, bulk refractive index and group velocity at frequency $\omega_{l}$, respectively. For a lossless medium, the dielectric constant $\epsilon_{l}(\mathbf{r})$ is real-valued and can be generally written as $\epsilon_{l}(\mathbf{r})=n_{l}^{2}+2 n_{l} \Delta n_{l}(\mathbf{r})$, where $\Delta n_{l}(\mathbf{r})$ is a small correction to the bulk refractive index $n_{l}$ that accounts for e.g. index guiding or cavity effects for the signal and/or idler fields, like in singly- or doubly-resonant OPOs. Equations (1) should be supplemented with the boundary conditions at the crystal (or cavity) boundaries. In an OPO geometry, a traveling-wave pump field shines on the crystal, and the signal and idler fields are spontaneously generated at the OPO instability as stationary outgoing waves. Time reversal of the underlying equations implies that, if $E_{l}(\mathbf{r})$ is a stationary solution to Eqs.(1) at the OPO instability point, then $E_{l}^{*}(\mathbf{r})$ is still a solution to the same equations. Since the complex conjugation replaces outgoing waves with incoming waves, the time-reversed process of OPO is the perfect annihilation of the incoming signal and idler fields, i.e. a colored CPA is realized. A schematic of the OPO instability and of its time-reversal is depicted in Fig.1. It should be noted that the present analysis is strictly valid only for classical fields, and phenomena involving few photons as well as quantum noise are not considered in such a semiclassical approach. If quantum fields are considered, spontaneous parametric down-conversion occurs, and the complete annihilation (conversion to the pump field) of both incident fields cannot be strictly reached.

Time-reversed backward OPO. In the following, we will focus our analysis to the backward OPO configuration in a QPM crystal of length $L$, which allows for mirrorless OPO [i.e. $\Delta n_{l}=0$ in Eqs.(1)]. In the plane-wave approximation [11, for a backward configuration the pump and signal fields copropagate along the positive $z$ direction, whereas the idler wave is counter-propagating along the negative $z$ direction, as shown in Fig.2(a). Phase matching of the interaction is ensured by a QPM grating $\chi^{(2)}(z+\Lambda)=\chi^{(2)}(z)$ with period $\Lambda$ satisfying the firstorder QPM condition $2 \pi / \Lambda=\left|k_{3}+k_{2}-k_{1}\right|$. After setting $E_{1,3}(z, t)=(1 / \kappa l)\left(\omega_{1,3} / n_{1,3}\right)^{1 / 2} a_{1,3}(x, t) \exp \left(-i k_{1,3} z\right)$, $E_{2}(z, t)=(1 / \kappa l)\left(\omega_{2} / n_{2}\right)^{1 / 2} a_{2}(z, t) \exp \left(i k_{2} z\right)$ for the forward- and backward-propagating waves and introducing the normalized spatial propagation distance $Z=z / L$, from Eqs.(1) the following coupled-mode equations can be obtained for the dimensionless envelopes $a_{l}$ after av-
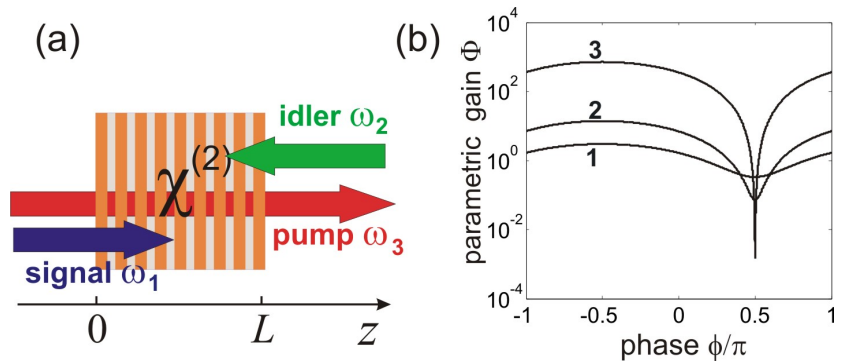

FIG. 2. (Color online) (a) Schematic of backward parametric interaction of pump, signal and idler waves in a nonlinear QPM crystal. (b) Behavior of the parametric gain $\Phi$ versus the phase difference $\phi$ of incident signal and idler fields for increasing values of the normalized input pump amplitude $a_{30}$ in the no-pump-depletion approximation and below the OPO threshold. Curve (1): $a_{30}=\pi / 6$; curve (2): $a_{30}=\pi / 3$; curve (3): $a_{30}=\pi / 2.1$. The dip at $\phi=\pi / 2$ is the signature of colored CPA.

eraging the rapidly-oscillating terms in $z$ [11, 15]

$$
\begin{aligned}
\pm \partial_{Z} a_{1,2}+\left(L / v_{1,2}\right) \partial_{t} a_{1,2} & =-i a_{2,1}^{*} a_{3} \\
\partial_{Z} a_{3}+\left(L / v_{3}\right) \partial_{t} a_{3} & =-i a_{1} a_{2}
\end{aligned}
$$

where $\kappa=\left(1 / 2 c_{0}\right)\left(\omega_{1} \omega_{2} \omega_{3} / n_{1} n_{2} n_{3}\right)^{1 / 2} \chi_{M}$ and $\chi_{M}$ is the Fourier amplitude of $\chi^{(2)}(z)$ at the spatial frequency $2 \pi / \Lambda$. Without loss of generality, $\kappa$ is assumed to be realvalued and positive. The intensities $I_{l}$ of the three waves are given by $I_{l}=\epsilon_{0} c_{0} n_{1} n_{2} n_{3} \lambda_{1} \lambda_{2} \lambda_{3}\left|a_{l}\right|^{2} /\left[2 \pi^{2} L^{2} \chi_{M}^{2} n_{l} \lambda_{l}\right]$, where $\lambda_{l}=2 \pi c_{0} / \omega_{l}$ are the (vacuum) wavelengths of the three waves $(l=1,2,3)$. To investigate the time reversal of OPO, we assume that a continuous-wave pump with amplitude $a_{3}(0) \equiv a_{30}$ is injected into the nonlinear crystal, together with two signal and idler waves with amplitudes $a_{1}(0)$ and $a_{2}(1)$, respectively.

Let us first assume that the amplitudes of injected signal and idler fields are much smaller than the pump amplitude $a_{30}$ (small-signal analysis). Then in the no-pumpdepletion approximation, $a_{3}(Z) \simeq a_{30}$, Eqs.(2) reduce to a system of two coupled linear equations for the amplitudes $a_{1}(Z)$ and $a_{2}^{*}(Z)$ [8], which yield the algebraic relation $\left(a_{1}(1), a_{2}^{*}(1)\right)^{T}=\mathcal{M}\left(a_{1}(0), a_{2}^{*}(0)\right)^{T}$ between the amplitudes of signal and idler fields at input $(Z=0)$ and output $(Z=1)$ crystal planes. Assuming without loss of generality a positive and real-valued amplitude $a_{30}$, the coefficients of the transfer matrix $\mathcal{M}$ read explicitly $\mathcal{M}_{11}=\mathcal{M}_{22}=\cos \left(a_{30}\right)$ and $\mathcal{M}_{12}=\mathcal{M}_{21}=-i \sin \left(a_{30}\right)$. The vanishing of $\mathcal{M}_{22}$ as the pump amplitude is increased from zero defines the mirrorless OPO threshold, which is attained at $a_{30}=\pi / 2$ (see, e.g., [8, 11]). It is worth observing that the transfer matrix $\mathcal{M}$ has the same structure as that encountered in the $\mathcal{P} \mathcal{T}$-symmetric CPA-laser device, recently proposed in Refs. 4, 5. In particular, since $\mathcal{M}_{11}=\mathcal{M}_{22}$, the simultaneous vanishing of $\mathcal{M}_{11}$ and $\mathcal{M}_{22}$ at the OPO instability threshold indicates that the system can simultaneously emits coherent signal and idler waves and acts as a colored CPA for appropriate excitation. This means that an OPO at the instability 
threshold basically realizes a kind of colored CPA-laser system. Like in the CPA-laser device, colored CPA requires coherent excitation of the crystal with signal and idler fields of appropriate amplitude and phase relationship, and the degree of absorption/amplification can be conveniently tuned by varying the relative phase of the two incoming beams. In fact, let us consider a pump level close to (but below) the threshold for OPO instability, and let us assume that the crystal is simultaneously illuminated, from opposite sides, by signal and idler waves with the same amplitude $A$ and phase difference $\phi$, namely let us assume $a_{1}(0)=A \exp (i \phi)$ and $a_{2}^{*}(1)=A$. The parametric gain for signal and idler waves after propagation in the crystal is given by $\Phi(\phi)=\left|a_{1}(1) / A\right|^{2}=$ $\left|a_{2}^{*}(0) / A\right|^{2}=\left\{\cos ^{2}(\phi)+\left[\sin (\phi)-\sin \left(a_{30}\right)\right]^{2}\right\} / \cos ^{2}\left(a_{30}\right)$. A typical behavior of $\Phi(\phi)$ versus the phase difference $\phi$ is shown in Fig.2(b) for increasing values of the pump level $a_{30}$. Note that, at $\phi=\pi / 2$ a dip is observed in the gain curve, with $\Phi(\pi / 2) \rightarrow 0$ as the threshold of OPO is approached. Such a dip is the clear signature of CPA. Far from $\phi=\pi / 2$, the system behaves conversely like a parametric amplifier $(\Phi>1)$, with a parametric gain that diverges as the OPO threshold is approached. The curves shown in Fig.2(b) clearly indicate that an optical parametric amplifier is capable of attenuating strongly as well when coherently illuminated from both sides, for an appropriate relative phase of the incoming fields. Such a result basically stems from the well-known phase-sensitivity of the frequency conversion process, which controls the flow of energy from the pump to the signal and idler waves.

The previous considerations are based on a small-signal analysis of Eqs.(2), which neglects pump depletion effects. However, a more comprehensive analysis should consider the full nonlinear regime, i.e. should account for pump depletion effects. Such an analysis is of major importance to check the stability of the CPA regime against the spontaneous emission of outgoing signal and idler waves. The exact solution corresponding to CPA in the nonlinear regime can be constructed following a similar procedure used in the theory of backward OPO [11, but with modified boundary conditions. Let us search for a stationary solution to Eqs.(2) of the form

$$
\bar{a}_{1}(Z)=a_{0}^{*} \sin [\theta(Z)], \quad \bar{a}_{2}(Z)=-i a_{0} \cos [\theta(Z)],
$$

where $a_{0}$ is a complex parameter and the angle $\theta(Z)$ is an unknown function. Substitution of Eq.(3) into Eqs.(2) yields the following coupled equations for $\bar{a}_{3}(Z)$ and $\theta(Z)$

$$
\partial_{Z} \theta=\bar{a}_{3}, \quad \partial_{Z} \bar{a}_{3}=-\left(\left|a_{0}\right|^{2} / 2\right) \sin (2 \theta) .
$$

The colored CPA regime is attained by imposing the boundary conditions $\bar{a}_{1}(1)=\bar{a}_{2}(0)=0$ for the signal and idler fields, and $\bar{a}_{3}(0)=a_{30}$ for the pump field. The former conditions can be satisfied by taking $\theta(0)=\pi / 2$ and $\theta(1)=n \pi$, where $n=1,2,3, \ldots$. Note that in this case $\left|a_{0}\right|$ corresponds to the normalized amplitudes of the counter-propagating signal and idler fields incident onto the crystal at the $Z=0$ and $Z=1$ facets, respectively. For an assigned pump level $a_{30}$, the value of $\left|a_{0}\right|$ has to be consistently determined from Eqs.(4) with the appropriate boundary conditions. In fact, from Eqs.(4) it follows that the angle $\theta(Z)$ satisfies the pendulum equation $\left(d^{2} \theta / d Z^{2}\right)+\left(\left|a_{0}\right|^{2} / 2\right) \sin (2 \theta)=0$, which admits of the constant of motion (energy) $E=(1 / 2)(d \theta / d Z)^{2}-$ $\left(\left|a_{0}\right|^{2} / 4\right) \cos (2 \theta)$. Hence the following relation holds

$$
\int_{\theta(0)=\pi / 2}^{\theta(1)=n \pi} \frac{d \theta}{\sqrt{2 E+\left(\left|a_{0}\right|^{2} / 2\right) \cos (2 \theta)}}=\int_{0}^{1} d Z=1 .
$$

The value of the energy $E$ is determined by the boundary condition at $Z=0$ and reads $E=(1 / 2) a_{30}^{2}+\left|a_{0}\right|^{2} / 4$. After setting $\eta \equiv\left|a_{0} / a_{30}\right|^{2}$, from Eq.(5) one readily obtains

$$
(\sqrt{1+\eta}) a_{30}=(2 n-1) K\left(\sqrt{\frac{\eta}{1+\eta}}\right)
$$

where $K(\xi) \equiv \int_{0}^{\pi / 2} d q /\left[1-\xi^{2} \sin ^{2}(q)\right]^{1 / 2}$ is the complete elliptic integral of the first kind. For a given order $n$, Eq.(6) implicitly defines the relation between $\left|a_{0}\right|$ and $a_{30}$, which is depicted in Fig.3(a) for the lowest-order $(n=1)$ state. Hence, to realize the CPA condition, two conditions should be satisfied: (i)the relative phase between signal and conjugate idler waves is fixed according to Eq.(3); (ii) for a given injected pump level $a_{30}$, the normalized signal and idler wave amplitudes injected into the crystal from the two sides should take the same value $\left|a_{0}\right|$ defined by the curve of Fig.3(a). In particular, for small values of $\left|a_{0}\right|$ the amplitude $a_{30}$ of the pump wave that realizes the CPA condition is exactly the threshold value $\pi / 2$ of OPO instability, according to the small-signal analysis previously discussed. The explicit behaviors of $a_{l}(Z)$ along the crystal are then obtained by solving Eqs.(4) and using Eqs.(3). Note that the phase of the amplitude $a_{0}$, which defines the absolute phases of the injected signal and idler fields, remains fully undetermined, so that if $\left\{\bar{a}_{1}(Z), \bar{a}_{2}(Z), \bar{a}_{3}(Z)\right\}$ is a stationary solution to Eqs.(2) corresponding to CPA, then $\left\{\bar{a}_{1}(Z) \exp (i \varphi), \bar{a}_{2}(Z) \exp (-i \varphi), \bar{a}_{3}(Z)\right\}$ is a solution as well for an arbitrary phase $\varphi$. As an example, Fig.3(b) shows the numerically-computed behavior of the normalized field intensities $\left|\bar{a}_{l}(Z)\right|^{2}$ for pump, signal and idler fields along the crystal for the lowest-order CPA solution $(n=1)$ corresponding to $\eta=5$ [point C of Fig.3(a)]. Note that the annihilation of the signal and idler photons corresponds to the creation of pump photons, rather than to the depletion as in the backward OPO [11. Higherorder $(n \geq 2)$ CPA solutions show oscillating power exchange among signal, idler and pump fields along the crystal. As an example, Fig.3(c) shows the behavior of $\left|\bar{a}_{l}(Z)\right|^{2}$ along the crystal for the $n=2$ mode corresponding to $\eta=5$.

The stability of the CPA solution can be investigated by a standard linear stability analysis [16. After setting $a_{l}(Z, t)=\bar{a}(Z)+\delta a_{l}(Z, t)$ in Eqs.(2), where $\delta a_{l}(Z, t)$ are small perturbations $(l=1,2,3)$, the most general solu- 

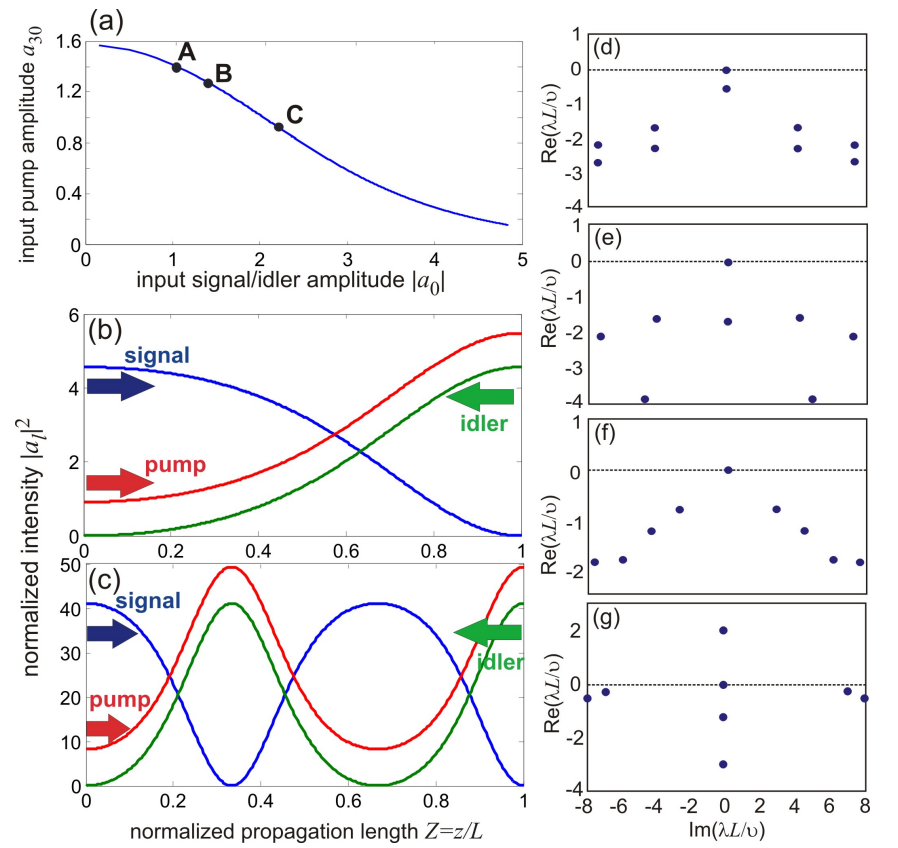

FIG. 3. (Color online) (a) Behavior of the input pump amplitude $a_{30}$ versus the amplitude $\left|a_{0}\right|$ of input signal and idler fields corresponding to the lowest-order CPA solution, numerically computed from Eq.(6) with $n=1$. The points A, B and $\mathrm{C}$ in the figure correspond to $\eta=0.5, \eta=1$ and $\eta=5$, respectively, where $\eta \equiv\left|a_{0} / a_{30}\right|^{2}$. (b) Behavior of the normalized intensities $\left|a_{l}(Z)\right|^{2}$ of pump, signal and idler fields inside the nonlinear crystal for the lowest-order $(n=1)$ CPA solution corresponding to $\eta=5$ [point C of Fig.3(a)]. (c) Same as (b), but for the second-order $(n=2) \mathrm{CPA}$ solution with $\eta=5$. (d-f) Loci of the eigenvalues $\lambda$ of the linearized equations for the lowest-order CPA solution corresponding to points A, B and $\mathrm{C}$ of Fig.3(a). (g) Same as (d-f), but for the second-order CPA solution of Fig.3(c). In the computation of the eigenvalues, the same group velocity $v_{1}=v_{2}=v_{3}=v$ has been assumed for the three waves.

tion to the linearized equations $\delta a_{l}(Z, t)$ is given by an ar- bitrary superposition of solutions of the form $\delta a_{l}(Z, t)=$ $u_{l}(Z) \exp (\lambda t)+v_{l}^{*}(Z) \exp \left(\lambda^{*} t\right)$, where the vector of perturbation amplitudes $\mathbf{v} \equiv\left(u_{1}, u_{2}, u_{3}, v_{1}, v_{2}, v_{3}\right)^{T}$ satisfies the linear system $(d \mathbf{v} / d Z)=\mathcal{G}(Z, \lambda) \mathbf{v}$ and $\lambda$ is a complex parameter whose real part determines the growth or decay rate of the perturbation. The explicit form of the $6 \times 6$ matrix $\mathcal{G}(Z, \lambda)$ is given in the supplementary material [16. The eigenvalues $\lambda$ are then determined after imposing the boundary conditions for the perturbations at the $Z=0$ and $Z=1$ planes [16. Owing to the phase invariance of the CPA solution, a neutrally stable mode with eigenvalue $\lambda=0$ is always found. An instability arises provided that the real part of at least one of the other eigenvalues is positive. Extended numerical simulations indicate that the lowest-order $(n=1) \mathrm{CPA}$ solution is stable at any pump level, whereas higher-order $(n \geq 2)$ CPA solutions are linearly unstable. As an example, Figs.3(d-f) show the numerically-computed loci of the eigenvalues $\lambda$ for the lowest-order $(n=1) \mathrm{CPA}$ solution at $\eta=0.5, \eta=1$ and $\eta=5$, respectively, corresponding to points A, B and C in Fig.3(a). Note that all the eigenvalues lie in the lower-half complex plane, and thus there are not unstable modes. Conversely, Fig.3(g) depicts the eigenvalues for the $n=2 \mathrm{CPA}$ solution at $\eta=5$, indicating the existence of an unstable mode associated to the eignevalue with a positive real part.

Conclusion. In this work, time reversal of optical parametric oscillation has been proposed and shown to realize a kind of "colored" coherent perfect absorber. A detailed analysis has been presented for the time reversal of mirrorless OPO. At threshold and in the no-pump-depletion limit, it has been shown that the OPO can simultaneously emit outgoing coherent signal and idler waves, and perfectly absorb incoming coherent signal and idler waves, thus realizing a kind of CPA-laser device [4, 5]. As compared to previous studies on CPA and CPA-laser systems [2 5], CPA operation has been extended here into the nonlinear regime. In particular, for the mirrorless OPO a proof of the stability of the colored CPA operation for the fundamental CPA mode has been presented.
[1] R.J. Potton, Rep. Prog. Phys. 67, 717 (2004).

[2] Y. D. Chong, Li Ge, Hui Cao, and A. D. Stone, Phys. Rev. Lett. 105, 053901 (2010); see also the viewpoint of S. Longhi, Physics 3, 61 (2010).

[3] W. Wan, Y. Chong, Li Ge, H. Noh, A.D. Stone, and Hui Cao, Science 331, 889 (2011).

[4] S. Longhi, Phys. Rev. A 82, 031801(R) (2010).

[5] Y.D. Chong, Li Ge, and A.D. Stone, Phys. Rev. Lett. 106, 093902 (2011).

[6] J.A. Armstrong, N. Bloembergen, J. Ducuing, and P.S. Pershan, Phys. Rev. 127, 1918 (1962).

[7] J.A. Giordmaine and R.C. Miller, Phys. Rev. Lett. 14, 973 (1965).

[8] A. Yariv, Quantum Electronics, 3rd ed. (Wiley, New York, 1988).

[9] M.H. Dunn and M. Ebrahimzadeh, Science 296, 1513
(1999).

[10] S. E. Harris, Appl. Phys. Lett. 9, 114 (1966).

[11] Y.J. Ding and J.B. Khurgin, IEEE J. Quant. Electron. 32, 1574 (1996).

[12] C. Canalias and V. Pasiskevicius, Nature Photon. 1, 459 (2007).

[13] G. Strömqvist, V. Pasiskevicius, and C. Canalias, Appl. Phys. Lett. 98, 051108 (2011).

[14] A. De Rossi and V. Berger, Phys. Rev. Lett. 88, 043901 (2002); M. Ravaro, Y. Seurin, S. Ducci, G. Leo, V. Berger, A. De Rossi, and G. Assanto, J. Appl. Phys. 98 98, 063103 (2005).

[15] S. Longhi, M. Marano, and P. Laporta, Phys. Rev. A 66, 033803 (2002).

[16] See the supplementary material 